\title{
IAC-08-A.6.2.6
}

\section{THE NASA ROBOTIC CONJUNCTION ASSESSMENT PROCESS: OVERVIEW AND OPERATIONAL EXPERIENCES}

\author{
Lauri Kraft Newman \\ NASA Goddard Space Flight Center \\ Greenbelt, MD, 20771, USA \\ lauri.k.newman@,nasa.gov
}

\begin{abstract}
Orbital debris poses a significant threat to spacecraft health and safety. Recent events such as China's antisatellite test and the Breeze-M rocket explosion have led to an even greater awareness and concern in the satellite community. Therefore, the National Aeronautics and Space Administration (NASA) has established requirements that routine conjunction assessment screening shall be performed for all maneuverable spacecraft having perigees less than $2000 \mathrm{~km}$ or within $200 \mathrm{~km}$ of geosynchronous altitude. NASA's Goddard Space Flight Center (GSFC) has developed an operational collision risk assessment process to protect NASA's high-value unmanned (robotic) assets that has been in use since January 2005. This paper provides an overview of the NASA robotic conjunction assessment process, including descriptions of the new tools developed to analyze close approach data and of the risk mitigation strategies employed. In addition, statistical data describing the number of conjunctions experienced are presented. A debris avoidance maneuver performed by Aura in June of 2008 is described in detail to illustrate the process
\end{abstract}

\section{$\underline{\text { BACKGROUND }}$}

Orbital debris poses a significant threat to spacecraft health and safety. The current number of catalogued objects is greater than 13,000, with the number of objects increasing by several hundred per year (Ref 1). Most of these tracked objects are characterized as orbital debris. Satellites routinely collide with small particles that cause little or no damage. However, if a large particle were to hit an operational satellite, the impact could result in the end of the mission. A large part of the orbital debris population resides in low earth orbit (LEO), where the density distribution of cataloged objects is concentrated near mean equatorial altitudes of $700-1100 \mathrm{~km}$, and in Geosynchronous (GEO) orbit. Recent events such as China's anti-satellite (ASAT) test and the Breeze-M rocket explosion highlight the importance of having a robust operations concept that includes monitoring, analyzing, and mitigating collision risks.

NASA has long required Conjunction Assessment (CA) for its manned assets. That process is implemented and operated by the NASA Johnson Space Center (JSC). However, CA was not being performed for the unmanned (robotic) on-orbit missions. In 2004, in response to the growing debris risk, NASA Goddard Space Flight Center (GSFC) developed and implemented a process for providing routine CA operations to protect the Morning and Afternoon Earth Science Constellations (ESCs) (Ref 2). These high-value assets reside in sun-synchronous frozen orbits with a $705 \mathrm{~km}$ mean equatorial altitude. Their mean local solar crossing times are established in a relationship that allows the missions to share their datasets and cross-calibrate their instruments. The 
missions are managed independently by several different NASA centers as well as the Centre National d'Etudes Spatiales (CNES) and the Comision Nacional de Actividades Espaciales (CONAE), but the mission operators work together to ensure the health and safety of the constellations. NASA JSC provided assistance in establishing the robotic process, which needed to be somewhat different from the manned process due to the different orbit regimes and different operations processes.

In August of 2007, because of the increasing threat posed by orbiting debris, NASA established a policy (NASA Procedural Requirement 8715.6A) that requires routine $\mathrm{CA}$ operations for robotic assets that have maneuvering capability and that have perigees lower than $2000 \mathrm{~km}$ or pass within $200 \mathrm{~km}$ of geosynchronous altitude (Ref 3). Since the signing of the policy, the need arose for a robust automated system that could easily provide support to missions in many orbit regimes. Under management of the Space Systems Protection Mission Support Office (Code 590.1), the GSFC $\mathrm{CA}$ process is now available to all NASA unmanned (robotic) missions. The process is currently being used to support 24 spacecraft in a variety of orbit regimes. In addition to the ESC missions, these include the Tracking and Data Relay Satellite System constellation, the Gamma Ray Large Area Space Telescope (GLAST), the Tropical Rainfall Measurement Mission (TRMM), JASON, and the Ocean Surface Topography Mission (OSTM)/JASON-2. This paper describes the NASA Robotic Conjunction Assessment Process and its application to customer assets.

\section{PROCESS OVERVIEW}

The NASA Robotic Conjunction

Assessment process consists of 3 steps:

1. Generating close approach predictions between customer assets and other objects in the United States Strategic Command's (USSTRATCOM) Space Object Catalog

2. Assessing the collision risk posed by predicted close approach events

3. Working with mission Owner/Operators to plan any necessary risk-mitigating action

The details for each step are described in this section.

\section{Step 1: Event Prediction Screening}

The first step is to predict the close approach events. USSTRATCOM is chartered with tracking objects in orbit around the Earth. Each tracked object is assigned a unique identification number and documented in the High Accuracy space object Catalog (HAC). USSTRATCOM can provide a service whereby they screen Owner/Operator ephemerides against the Catalog and provide predicted miss distance data. In order to obtain that information, NASA entered into an agreement with the Department of Defense under which USSTRATCOM provides support to the GSFC Space Systems Protection Mission Support Office CA Team by performing routine screenings for customer missions against the HAC (Ref 4). Per this agreement as well as Reference 5, a dedicated Orbital Safety Analyst (OSA) provides conjunction 
assessment data for NASA customers. The OSA computes separation distances between each of the catalogued objects and the customer ephemerides provided by the mission Owner/Operator. The nominal prediction frequency is once a day Monday through Friday, with screenings against the full HAC on Monday, Wednesday, and Friday and supplemental screenings for interesting events on Tuesday and Thursday. Predictions are made 7 days into the future for low-earth orbiting (LEO) assets and 10 days into the future for geosynchronous (GEO) assets. Any planned maneuvers are modeled in the ephemerides provided by the mission.
Three different mission safety volumes are used in the screening process to identify predicted conjunction events. These volumes are defined centered on the asset. If a secondary object is predicted to intersect the safety volume, the volume is violated and a specified action is taken. The safety volumes dictate different data product deliveries as well as actions taken by the CA Team. Table 1 lists the dimensions of the safety volumes that have been defined for the NASA robotic CA process. The coordinate frame for all volumes is the radial, in-track, crosstrack (RIC) coordinate frame.

Table 1: Safety Volume Definitions

\begin{tabular}{|c|c|l|c|c|c|}
\hline & \multicolumn{3}{|c|}{ LEO Safety Volumes } & $\begin{array}{c}\text { GEO Safety } \\
\text { Volumes }\end{array}$ & Data Product \\
\hline \hline & $\begin{array}{c}\text { Radial } \\
(\mathrm{km})\end{array}$ & $\begin{array}{l}\text { In-Track } \\
(\mathrm{km})\end{array}$ & $\begin{array}{c}\text { Cross- } \\
\text { Track }(\mathrm{km})\end{array}$ & $\begin{array}{c}\text { Stand-off } \\
\text { Radius } \\
(\mathrm{km})\end{array}$ & \\
\hline \hline $\begin{array}{c}\text { Monitor } \\
\text { Volume } \\
(\text { ellipsoid) }\end{array}$ & \pm 2 & \pm 25 & \pm 25 & 40 & $\begin{array}{c}\text { Conjunction } \\
\text { Assessment } \\
\text { Screening } \\
\text { Summary }\end{array}$ \\
\hline $\begin{array}{c}\text { Tasking/Alert } \\
\text { Volume } \\
\text { (box) }\end{array}$ & \pm 0.5 & \pm 5 & \pm 5 & 15 & $\begin{array}{c}\text { Orbital } \\
\text { Conjunction } \\
\text { Message }\end{array}$ \\
\hline $\begin{array}{c}\text { Watch } \\
\text { Volume } \\
\text { (sphere })\end{array}$ & $1 \mathrm{~km}$ stand-off radius & 2 & $\begin{array}{c}\text { Vector } \\
\text { Covariance } \\
\text { Message }\end{array}$ \\
\hline
\end{tabular}

The Monitor Volume is the largest safety volume and serves as the initial reporting filter. For each asset, all objects that are predicted to violate the Monitor Volume are reported to the GSFC CA Team via the Conjunction Assessment Screening Summary. This product is provided each time the catalog screening is performed and includes the catalog identification number of the secondary object and the RIC miss distance components at the time of closest approach. The Tasking Volume is a smaller volume, and close approach predictions that fall within this volume require further analysis. The OSA will examine the orbit determination solution for both objects and request additional tracking on the secondary object if necessary so that a more accurate orbit can be determined. For each Tasking Volume violation, state vector and state 
vector uncertainty information at the time of closest approach is provided to the GSFC CA Team in the form of an Orbital Conjunction Message (OCM). This information allows for the collision probability to be calculated. Additional orbit determination details such as the fit-span, number of observations, and solve-for parameters at the time of closest approach are also included in the OCM. For all watch volume violations, the OSA provides a Vector Covariance Message (VCM) for each object. The VCM contains state and covariance information at the epoch of the orbit determination solution, allowing the state to be propagated by the GSFC CA Team for further analysis.

\section{Step 2: Risk Analysis}

The second step is the processing of the screening data. The GSFC CA Team has developed and implemented a set of tools called the Collision Assessment System (CAS) which is used to generate reports and perform event evaluation. The CAS consists of the following modules:

- Secure File Transfer Protocol (SFTP)

- Parser/Monitor Scripts

- Secure Access Database

- Collision Assessment and Mitigation (CAM) Tool Suite:

1. Conjunction Visualization Script

2. 2-D Collision Probability Utility

3. Monte Carlo Collision Simulation

4. Nonlinear Collision Probability Tool

5. Time History Trending Tool

\author{
6. Collision Avoidance \\ Planning Module \\ - Secure Webpage (Portal) \\ - Configuration Management \\ System
}

The portion of the CAS that provides the set of tools used to analyze close approach events is known as the Collision Assessment and Mitigation (CAM) tool suite. The CAM tool suite is built using the Commercial-Off-TheShelf (COTS) software products FreeFlyer $^{\circledR}$ (FF) and Matlab ${ }^{\circledR}$. The tool suite interfaces to the Secure Access Database for data input and output. Some of the tools are run autonomously as part of the routine daily CA processing; all may be run manually to further assess the risk posed by a conjunction event. Further details concerning the architecture and operation of the CAS may be found in References 6 and 7.

Each time data is received from the OSA, the GSFC CA team is responsible for processing the data and providing risk assessment analysis results to the mission stakeholders. Parser/Monitor scripts run constantly looking for new data. Once a delivery is detected, the data is placed into the CA Database and the CAS begins analyzing the data, an automated process through which a Conjunction Summary Report is automatically generated and distributed via e-mail to mission stakeholders. Each mission receives a customized Conjunction Summary Report which may contain data for multiple assets. The report echoes the screening results (miss distances and time of closest approach) computed by the OSA for the Owner/Operator ephemeris and the JSpOC solution. It also contains values computed by the CAS, including 
Probability of Collision $\left(\mathrm{P}_{\mathrm{c}}\right)$ and clock angle. Event trends are plotted for the miss distance components, total miss distance, $\mathrm{P}_{\mathrm{c}}$, and clock angle. The report also lists any expected upcoming spacecraft maneuvers. In order to help analysts keep track of activities involving all spacecraft in a constellation, planned maneuvers and close approach predictions are listed on a 'CA Calendar' included in the report. The CA Calendar contains the following:

- Close approach predictions that are less than $1 \mathrm{~km}$

- Close approach events that have $\mathrm{P}_{\mathrm{c}}$ values greater than 1e-7

- Planned maneuver dates and start times

Mission stakeholders usually receive the Conjunction Summary Report less than an hour after the screening data is received by the CAS.

The second activity that the automated software performs is to analyze each OCM that was received and create an analysis package called the OCM Analysis Report. These reports are produced for each Tasking Volume event and posted to the CA Portal webpage. The OCMs are first ingested into the database, then each OCM is automatically processed through the $2-D$ Collision Probability Utility and the Monte Carlo Collision Simulation Tool. This processing produces a consolidated OCM Analysis Report package containing a series of plots and text reports which is posted to the Portal webpage for review by the analyst. The output from the 2-D Collision Probability Utility consists of the miss distance, various collision probability calculations, conjunction geometry information, and collision probability sensitivity analysis. The numerical results from the two different $\mathrm{P}_{c}$ computation utilities are placed back into the CAS database. This analysis occurs overnight for routine operations, since the data is received at the close of business. These reports are made available to the mission Owner/Operator upon request for high interest events, but are mainly used by the CA Team to analyze the event to determine the associated threat.

In the morning, the CA Team analyzes each OCM Analysis Report as well as accompanying data in the Screening Summary file to identify which close approach events pose a potential threat and thus warrant further analysis. In particular, the total miss distance and the R-I-C components as well as the probability of collision are considered. If after this inspection the item is still considered a potential threat, the analyst refers first to the orbit determination data located in the OCM. The length of the orbit solution fit, the number of tracks and observations available, as well as the number of observations used in the orbit solution are reviewed. The number and location of the stations that are tracking the secondary object are considered. With this information, the analyst then determines if it is necessary to contact the OSA and discuss potential changes to the tracking and orbit solutions. If all of the tracking observations for the secondary object are found to be from a point in the orbit not near the conjunction or if the object has not been tracked recently, a request is made of the OSA to try to obtain additional tracking data for the object. Requesting increased tasking does not guarantee that additional data will be received. 
Based on their evaluation of the data, the CA Team produces a "watchlist" detailing the response being taken for each event. Events are either classified as "not a threat", "monitor", or "threat". If the event is "not a threat", no further action is taken by the team. Items may be classified as "not a threat" because the conjunction geometry is such that the two items will not cross paths despite being close to each other, for instance. Items labeled as "monitor" are those which may not have credible orbit determination solutions. Time will be spent attempting to gather more data and further evaluate the event. As additional data is received, the CA Team continues to review the standard reports, but also begins to perform close approach event trending by 'linking' several solutions together in order to establish future trends. The Time History Trending Tool allows the engineer to plot time histories of parameters obtained from a series of OCMs. Data such as the miss distance, orbital elements, uncertainties, force model parameters, event time, and conjunction geometry can be trended and examined for consistency. The solution consistency is examined using RIC corrections at the time of closest approach (TCA) for both the primary and the secondary object ( $3 \sigma$ check) and a projected covariance plot in the conjunction plane. It is expected that as the time to closest approach shortens, the covariance on well-tracked objects should decrease. Also, it is expected that successive updates to the orbit determination solutions, and hence updates to the miss distance, should be consistent with the uncertainty represented by the covariance matrix. The CA Team prefers to see three consecutive consistent solutions in order to recommend a course of action based on the data.

Another criterion is that the $\mathrm{P}_{c}$ must be high and predicted to stay high. Several tools are used to predict the behavior of the $\mathrm{P}_{\mathrm{c}}$, such as how the collision probability value will evolve as the position uncertainty changes, which is called " $\mathrm{P}_{\mathrm{c}}$ forecasting". If the $\mathrm{P}_{\mathrm{c}}$ is high and the orbital uncertainties in the secondary object are large, chances are the $\mathrm{P}_{\mathrm{c}}$ will drop as the time to TCA gets shorter. Sometimes the secondary object is not well tracked. In these cases, the probability metric may be mathematically "high" due to the large uncertainty, but the true risk is difficult or impossible to quantify. Within the 2$D$ Collision Probability Utility, the value for $\mathrm{P}_{\mathrm{c}}$ is plotted for various scale factors applied to the covariance. Scale factors less than 1 represent a contraction of the covariance, which is expected as time to TCA decreases. If the $\mathrm{P}_{\mathrm{c}}$ is forecasted to go down with increased orbit determination accuracy, the event may not be a threat. If the $\mathrm{P}_{c}$ is forecasted to go up or stay static, the event may be a threat.

Finally, the conjunction geometry is examined. The Conjunction Visualization Script allows the CA Engineer to investigate the 3dimensional geometry of the encounter. In addition to providing time histories of the assets' orbital elements, the relative orbital geometry and 2-D and 3-D orbit visualizations are available. The Visualization Script is used to analyze the respective orbits of both objects. Time history orbital element information is generated and used to examine how the close approach event evolves over time; the close approach geometry is 
visually represented. The angle between the primary axis of the combined covariance in the conjunction plane, which is a two-dimensional surface that is perpendicular to the relative velocity vector, and the miss vector is also determined and is called the "clock angle". Investigation of this "clock angle" gives insight into how changes in the conjunction geometry will affect the $\mathrm{P}_{\mathrm{c}}$. For clock angles near zero, the $\mathrm{P}_{\mathrm{c}}$ is maximized for a given miss distance and a given covariance. Changes in conjunction geometry that drive the angle away from zero can be expected to decrease the $P_{c}$. There have also been cases where the miss distance is so small that any rotation of the combined covariance in the conjunction plane will not lead to any significant reduction in the $\mathrm{P}_{\mathrm{c}}$. In these cases, the conjunction plane plot tells you that future changes in the orbit determination solutions will not significantly alter the $\mathrm{P}_{\mathrm{c}}$.

Items considered to be a "threat" are those for which avoidance maneuver planning will likely be initiated as the TCA nears. Events for which maneuver planning is initiated are called "High Interest Events" (HIE). The support process for items classified as "threat" or HIE involves intensified conjunction screening and additional analysis prior to and following the event until either the threat goes away or a mitigation plan is executed. For high interest events, the GSFC CA Team produces a CA Summary Package (Power Point presentation package) that includes event trends, probability sensitivity analysis, and post-maneuver close approach predictions. The package is updated each time new data is received, and meetings are held with the Owner/Operator to discuss these updates. Recommendations made to the project are also included. If a Risk Mitigation Maneuver (RMM) is executed, a memo is also prepared and provided to the mission stakeholders to officially document the event. The memo is similar in content to the Power Point package.

\section{Step 3: Mitigation Planning}

Step 3 of the process is risk mitigation planning. When an event is considered to be a "threat", the Owner/Operator is notified by the CA Team, and the two groups work together to decide what approach will be taken to investigate possible mitigation options. Typically, the CA Team provides a "first guess" of the type, size, and timing for a maneuver that would mitigate the risk. In general, the CA Team usually chooses an opportunity during a day shift roughly 24 hours prior to the TCA. This allows enough time to gather as much information as possible about the conjuncting object while also allowing time following the maneuver for alongtrack differences to accumulate to mitigate the conjunction. Maneuver strategies, however, are ultimately dependent on individual mission orbit requirements and propulsion capability. The Flight Operations Team therefore provides input on mission constraints. These include limitations in the direction of the burn, the time of day the team likes to perform maneuvers based on operations procedures and constraints, and limits on the size of the burn necessary to maintain orbit requirements. Another factor that would contribute to the selection of a maneuver time is the availability of communications passes. Often the team attempts to schedule the maneuver 
during an already-scheduled pass to minimize the need for requesting additional services. Maneuver planning begins several days prior to the TCA, with daily checks to see whether to continue the planning or if the threat has mitigated itself. Assuming the threat is detected 5-7 days prior to the TCA, this process allows sufficient time to work the issue. Obviously, the maneuver planning process can be very complicated for a mission having many constraints. For instance, the Terra, Aqua, and Aura missions in the ESC are restricted to only performing orbitraising maneuvers, not orbit-lowering ones. They also have tight ground track control requirements that limit the amount that the orbit can be raised, depending on where the spacecraft is located within the control box at the time. Since planning time is usually limited, serially investigating multiple maneuver options and then rejecting them becomes a problem. The CA Team has developed a tool which allows the available trade space to be analyzed in advance, so that only one or two options need be considered (Ref 8). Sample output from the tool and a description of how it can be used to enable maneuver choice is described in the Aura case study below.

Once the maneuver option is chosen and the details are planned, an ephemeris is created and sent to the OSA for screening to make sure that no new conjunctions have been created by maneuvering. As updates continue to be received on the orbit determination solution for the conjuncting object, the plan is revisited at least daily to update the maneuver size, although the timing usually remains fixed due to comm. constraints. These updates will also be screened by the OSA. If the event is deemed no longer a threat, the maneuver planning is ended and no maneuver is executed. If the threat remains at a point in time when a final decision is required based on the operations concept for the spacecraft, the maneuver is executed.

The following example illustrates the process for the Aura versus 1399 event in June 2008.

Step 3: Operational GSFC CA Process Case Study - Aura Versus 1399

On Saturday the $21^{\text {st }}$ of June, 2008, the routine daily screening report identified a close approach between Aura and debris object 1399 (TRIAD 1 debris). The TCA was reported to be at 15:34 Z on the $27^{\text {th }}$ of June. The first reported miss distance on Monday was approximately $29.8 \mathrm{~m}$ with a high collision probability of $7.91 \mathrm{e}-2$. The CA Team evaluated the event over several days of increased tasking. While monitoring the event, the miss distance remained small and the $P_{c}$ remained high. These consistent solutions, coupled with the fact that the conjunctor was a well-tracked object receiving several new tracks each day from two different stations, led to high confidence in the predictions. The Aura Flight Project was notified and avoidance maneuver planning was initiated on Monday afternoon.

Available maneuver planning options were limited due to the ground track control requirements. Aura had recently performed a nominal drag make-up (DMU) maneuver, placing the spacecraft near the top of its control box. Any maneuver longer than two seconds in duration (roughly $0.01 \mathrm{~m} / \mathrm{s}$ in maneuver magnitude) would violate the orbit 
requirements, an option only to be undertaken as a very last resort. Fortunately, a maneuver of this size would mitigate the conjunction. However, there was significant concern about potential maneuver modeling inaccuracy, since Aura had never performed such a small maneuver. Since the orbit determination solution was so consistent and predictable, the CA Team recommended planning the maneuver for 48 hours prior to the TCA to allow sufficient time for along-track differences to accumulate and mitigate the conjunction regardless of large maneuver performance uncertainty. The Owner/Operator chose instead to perform the maneuver at TCA-24 hours hoping that the conjunction uncertainty would decrease, causing the $\mathrm{P}_{\mathrm{c}}$ to also decrease with constant miss distance predictions.

Figure 1 shows the maneuver trade space available for the Aura versus 1399 event. This tool is used to show the trade off between maneuver magnitude and phase time (the time between the maneuver execution and the TCA) and the effects on the post-maneuver miss distance. The tool assumes that posigrade maneuvers linearly increase the alongtrack uncertainty, as documented in Ref 8. The contour lines represent lines of constant post-maneuver miss distance at TCA. The burn size (delta-v) and phasing time can be located on the plot to find combinations which result in a miss distance that sufficiently mitigates the risk. For this event, the postmaneuver miss distance needed was at least 500 meters. Because the Aura mission also has a ground track control requirement, the green curve (read on the right hand vertical axis) shows the ground track error as a function of burn duration. The horizontal, dashed green lines indicate the limits of the Aura WRS-2 ground track control box of $18 \pm$ $10 \mathrm{~km}$. This tool aids in maneuver planning that not only mitigates risk imposed by the conjunction, but also satisfies operational constraints. The red dotted vertical line indicates the chosen burn duration, which is slightly less than the maximum allowed by the ground track control requirement. The star indicates the chosen burn duration and phasing time. The star shows that a burn duration of about $0.009 \mathrm{~m} / \mathrm{s}$ executed about 24 hours prior to TCA will result in a predicted post-maneuver miss distance of about $650 \mathrm{~m}$. Figure 1 also shows that this maneuver combination will not violate the ground track control requirement and results in a WRS-2 turnaround of $11 \mathrm{~km}$, well above the $8 \mathrm{~km}$ requirement.

Concurrent with risk mitigation maneuver planning, the GSFC CA team continued to monitor the evolution of the conjunction. Additional orbit solutions were requested from the OSA, and in all 10 solutions were received by Wednesday, June 25. The miss distance remained low and the probability of collision remained high, as the orbit determination solutions for the conjuncting object were consistent. The Wednesday afternoon solution reported the lowest miss distance of $13 \mathrm{~m}$ and the highest collision probability of $4.7 \mathrm{e}-1$ during the evolution of the conjunction event. As expected by the CA Team, the conjunction uncertainty did not unexpectedly decrease, and the maneuver was performed on Thursday morning.

Figures 2 shows the evolution of the miss distance of the close approach 
event throughout the week. The values shown in the graphs were computed at the TCA. The miss distance increased by $150 \mathrm{~m}$ between the first and second solutions, then began a steady trend downward which continued throughout the event, reaching a minimum value of $13 \mathrm{~m}$ prior to the execution of the risk mitigation maneuver on the morning of Thursday, June 26. The data points in these plots represent discrete solutions.

Figure 3 shows the $\mathrm{P}_{\mathrm{c}}$ time history trend. The graph shows that the probability remained extremely high throughout the event. Typically, if the miss distance predictions remain constant, the $\mathrm{P}_{\mathrm{c}}$ will drop as the propagation time and the position uncertainties decrease. However, since the miss distance decreased as the event evolved, the $\mathrm{P}_{\mathrm{c}}$ value remained high.

The final solution in Figures 2 and 3 is a post-maneuver definitive solution. This definitive solution is generated by collecting sufficient post-maneuver data and anchoring the OD epoch at TCA. The post-maneuver definitive miss distance was $795 \mathrm{~m}$, compared to the prediction of $819 \mathrm{~m}$. Both the predicted and definitive post-maneuver $\mathrm{P}_{\mathrm{c}}$ values were zero.

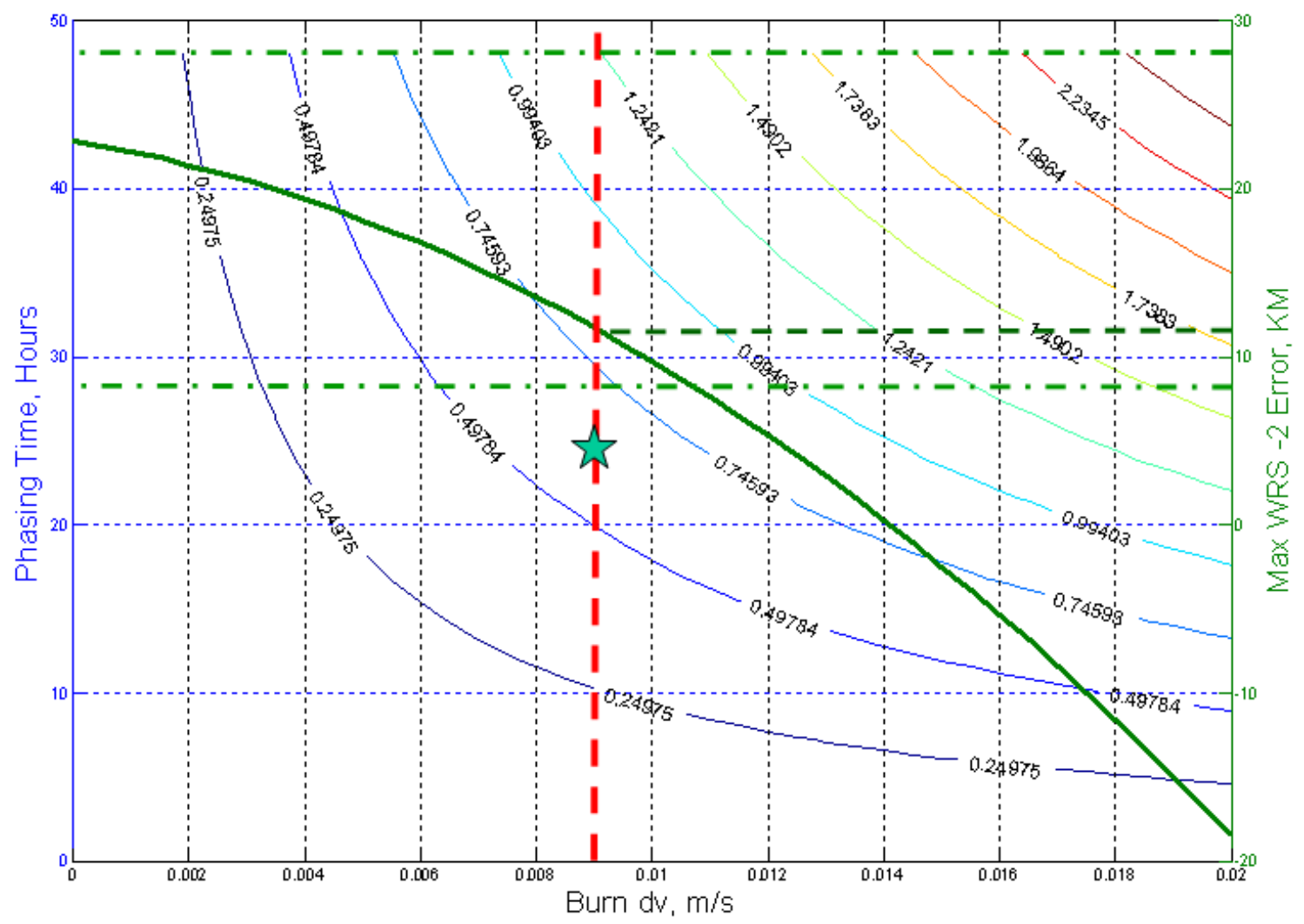

Figure 1: Aura vs. 1399 Maneuver Planning Trade Space (Ref 9) 
IAC-08-A.6.2.6

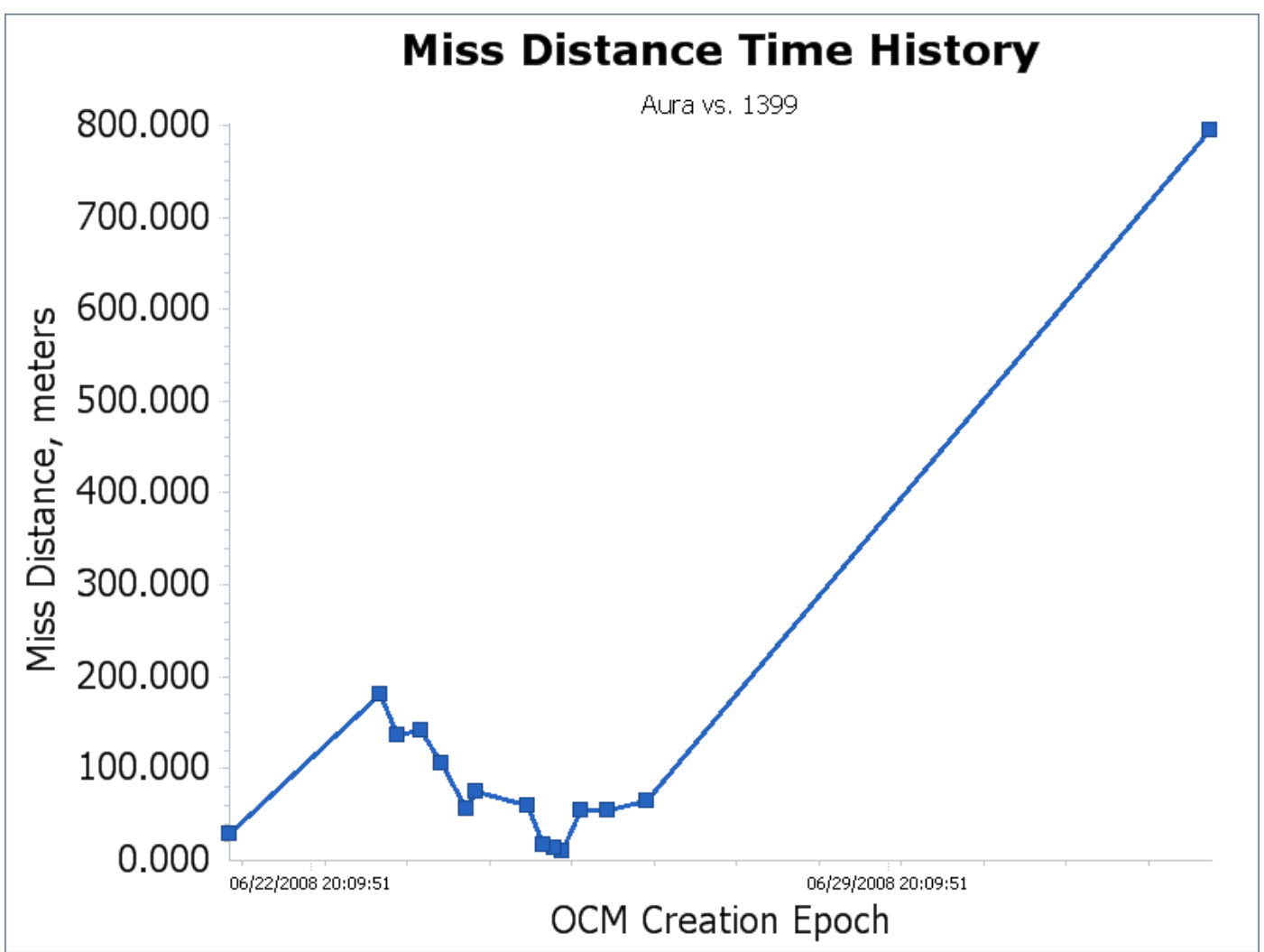

Figure 2: Aura Versus 1399 Miss Distance History (Ref 9)

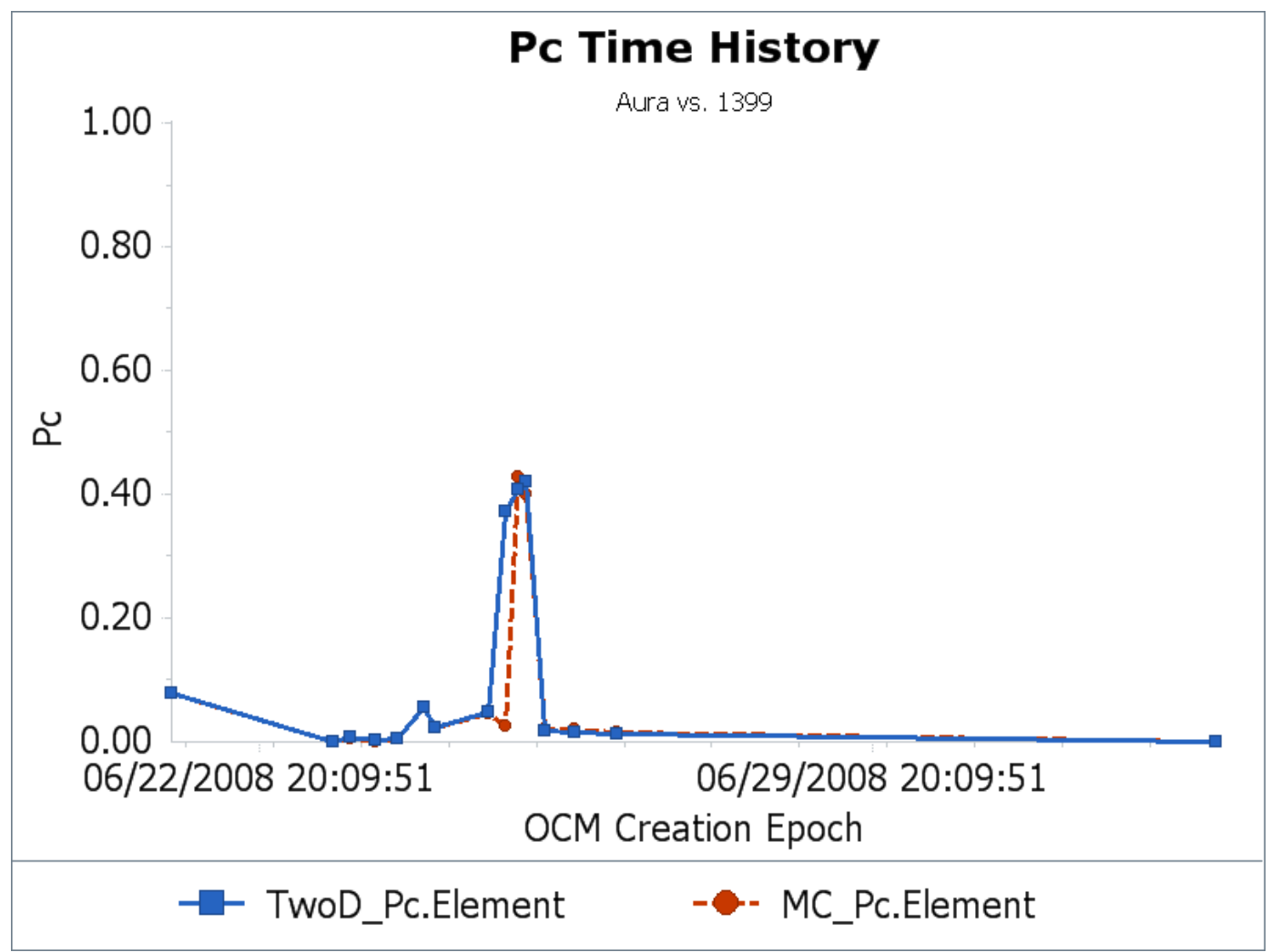

Figure 3: Aura Versus 1399 Collision Probability History (Ref 9) 


\section{IAC-08-A.6.2.6}

\section{STATISTICS AND OPERATIONAL EXPERIENCES}

This Conjunction Assessment risk evaluation process has been used in routine spacecraft operations at GSFC since January 2005. In addition to analyzing and assessing each conjunction event, the GSFC CA Team has been monitoring statistics reflecting the debris environment experienced by its customer's assets, especially the ESC missions. The database into which the OCM data is placed can be mined to discern trends in debris quantity, repeat conjunctions with the same debris, and other meaningful statistics that help the Team to refine and augment the process. Figure 3 shows the number of unique safety volume violations experienced by the ESC missions each month since screening began. The Figure shows that the constellation as a whole experiences about 750 Monitor Volume violations per month, which corresponds to about 15 per week per spacecraft. Assets average about 1 conjunction per week within the Tasking Volume, and about 3 High Interest Events per year.

As a result of implementing the process, there have been 7 times when a spacecraft has maneuvered to mitigate the risk posed by a conjunction event. Table 2 lists the spacecraft involved in these events, in addition to the minimum total miss distance and corresponding $\mathrm{P}_{\mathrm{c}}$ for that event. In addition to events which resulted in avoidance maneuvers, there were also four instances in which a planned orbit maintenance maneuver was waived off to avoid worsening the effects of a conjunction predicted shortly after the maneuver time.
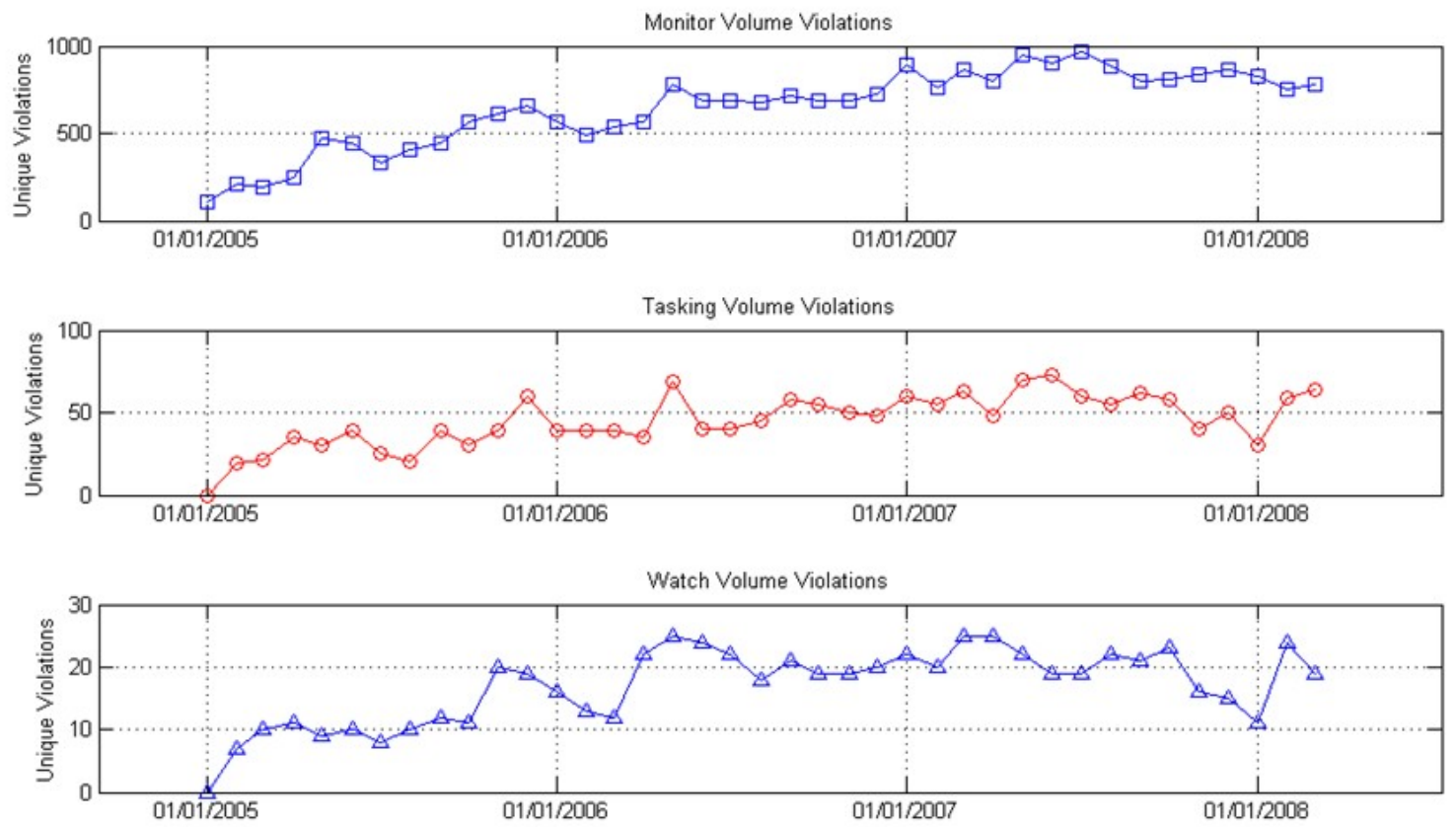

Figure 3: ESC Monthly Safety Volume Violations 
IAC-08-A.6.2.6

Table 2: Risk Mitigation Maneuvers Performed by the ESC

\begin{tabular}{|c|c|c|c|c|}
\hline Asset & Secondary & Maneuver Date & $\begin{array}{c}\text { Minimum Total } \\
\text { Miss (m) }\end{array}$ & $\mathbf{P}_{\mathbf{c}}$ \\
\hline Terra & $\begin{array}{c}14222 \\
\text { (SCOUT G-1) }\end{array}$ & 21-Oct, 2005 & 37 & $6.82 \mathrm{E}-2$ \\
\hline PARASOL & $\begin{array}{c}81257 \\
\text { (Analyst SAT) }\end{array}$ & 16-Jan, 2007 & 43 & $1.51 \mathrm{E}-3$ \\
\hline SAC-C & $\begin{array}{c}14345 \\
\text { (SL-8 DEB) }\end{array}$ & 16-Feb, 2007 & 57 & $3.40 \mathrm{E}-6$ \\
\hline Terra & $\begin{array}{c}31410 \\
\text { (FENGYUN 1C } \\
\text { DEB) }\end{array}$ & 22-Jun, 2007 & 18 & $1.58 \mathrm{E}-1$ \\
\hline CloudSat & $\begin{array}{c}28893 \\
\text { (SINAH 1) }\end{array}$ & 04-Jul, 2007 & 38 & $2.24 \mathrm{E}-2$ \\
\hline Aura & $\begin{array}{l}1399 \\
\text { (TRIAD 1 debris) }\end{array}$ & 26-Jun, 2008 & 11 & $4.80 \mathrm{E}-1$ \\
\hline CloudSat & $\begin{array}{c}8542 \\
\text { (Delta I Debris })\end{array}$ & 20-Jul, 2008 & 90 & $1.77 \mathrm{E}-3$ \\
\hline
\end{tabular}

IMPACT OF THE CHINESE ANTISATELLITE TEST

On January 11, 2007, the Chinese performed a test of an Anti-Satellite (ASAT) weapon during which they destroyed their Fengyun 1C weather satellite. This single event significantly impacted the debris environment near the ESC altitude, since the orbit of Fengyun 1C was $861 \mathrm{~km}$, only $150 \mathrm{~km}$ above the ESC and in a similar orbit plane. Over 2000 objects have been cataloged as a result of this event, when the debris environment usually increases by only about 200 objects per year (Ref 10). The first close approach between an ESC asset and a piece of Fengyun 1C debris occurred on Feb 4, just a few weeks after the event. Initially, ASAT debris made up 10 percent of the monthly predicted conjunctions. That average percentage has grown to $13 \%$ over the last 18 months. Figure 4 shows the monthly percentage of safety volume violations attributable to the ASAT debris. 


\section{IAC-08-A.6.2.6}
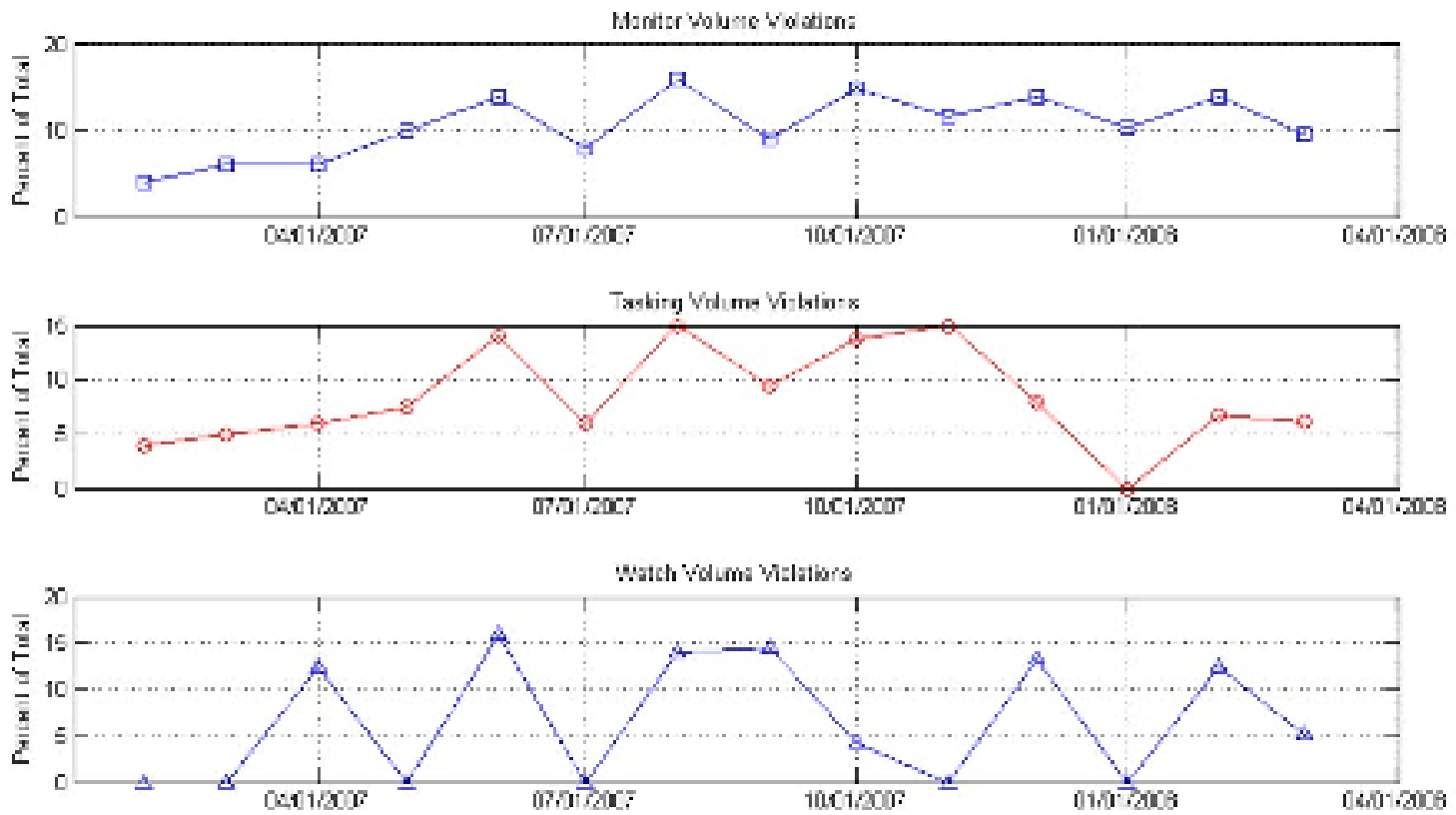

Figure 4: Percent of ESC Safety Volume Violations due to ASAT Debris

The GSFC CA Team has performed analysis to determine the longevity of the ASAT debris cloud. Refs $11 \& 12$ describe the details of that analysis. Ref 12 shows that in 20 years $70 \%$ of the catalogued Fengyun debris still remains, and that up to $20 \%$ of the debris remains on orbit 125 years from now. Since most of the debris is above the LEO altitudes used by the NASA robotic assets, the risk to those assets will continue to increase in the foreseeable future. In response, the GSFC CA Team is attempting to streamline its event evaluation process to ensure that appropriate risk mitigating action can be taken in minimum time.

\section{SUMMARY AND FUTURE PLANS}

The NASA Robotic Conjunction Assessment process has enabled the protection of many assets during the past four years. The automated process has enabled efficient support of many events, yet experience has shown that having a knowledgeable person experienced in orbit determination on the team to evaluate the orbit solutions for conjuncting objects is a necessity. In addition, each close approach event analyzed was found to be sufficiently unique that the approach of implementing a fixed decision-making criterion such as maneuvering any time the $P_{c}$ is a certain value was not taken. In the future, the CA Team plans to continue to look for ways to streamline the assessment process to be able to provide efficient support for multiple conjunctions simultaneously in anticipation of the growth in the number of events predicted to occur. Future work will include adding a probabilistic risk assessment component to the maneuver planning process.

\section{ACKNOWLEDGEMENT}

The author would like to acknowledge contributions of a.i. solutions employees Ryan Frigm, David McKinley, and Brian 


\section{IAC-08-A.6.2.6}

Robinson of the GSFC CA Team. They performed the analysis for the items described herein and also offered their help and advice.

\section{REFERENCES}

1. Liou, J., and Johnson, N.L., "Risks in Space from Orbiting Debris" Science Volume 311, 20 January 2006

2. Newman, Lauri K. and Matthew Duncan, "Establishment and Implementation of a Close Approach Evaluation and Avoidance Process for Earth Observing System Missions," AAS/AIAA Astrodynamics Specialist Conference, Keystone, CO, AIAA2006-6291.

3. NASA Office of Safety and Mission Assurance, "NASA Procedural Requirement for Limiting Orbital Debris Generation", NPR 8715.6A, $2 / 5 / 08$.

4. Memorandum of Agreement between the Department of Defense and the National Aeronautics and Space Administration.

5. Operating Instruction for Robotic Mission Support.

6. McKinley, D., "Conjunction Assessment System Architecture and Design Document," Technical Memorandum FDF-209-038, 6 July 2006.

7. McKinley, D., "Flight Dynamics (FD) Conjunction Assessment and Mitigation Tool Suite User's Guide," MOMS-FDUG-0246, 30 June 2006.

8. McKinley, David, "Maneuver Planning for Conjunction Risk Mitigation with Groundtrack Control Requirements," AAS 08-242, AAS/AIAA Astrodynamics Specialist Conference, Galveston, TX, Jan 2008.

9. Robinson, B., Frigm, R., "OCM

Analysis: Aura vs. 01399; TCA: 27Jun-2008 15:34:55 Z. Status Update: (Command Authorization Meeting) 25Jun-2008 20:00 Z (4 PM ET)," CA Summary Package briefing materials.

10. Johnson, M., Stansbery, E., Liou, J., Horstman, M., Stokley, C., Whitlock, D., "The Characteristics and
Consequences of the Break-Up of the Fengyun-1C Spacecraft" IAC-07A6.3.01.

11. Duncan, Matthew and David K. Rand, "Close Approach Prediction Analysis Of The Earth Science Constellation With The Fengyun-1c Debris," AAS 08-125, AAS/AIAA Astrodynamics Specialist Conference, Galveston, TX, Jan 2008.

12. Duncan, M., Rand, D., Wysack, J., "Long Term Trends of the Fengyun-1C Debris," a.i. solutions Technical Memorandum as delivered to General Dynamics Advanced Information Systems, 3 July 2008. 